\title{
Facilitating the Sharing of Patient Information Between Health Care Providers
}

\author{
Kieran Moore and Paul Belanger \\ KFL\&A Public Health, Kingston, ON, Canada
}

\section{Objective}

To describe how the South Eastern Integrated Information Portal (SHIIP) will support the Health Links program with the delivery of care for patients, by facilitating reporting, performance monitoring and quality improvement efforts. The portal-based technology that SHIIP uses to integrate all of a patient's clinical care information into summarized data and to provide real-time feedback will also be explained.

\section{Introduction}

In the current state of the health care system there is uneven access to primary care, and too many people struggling to navigate the system are receiving care in the hospital for issues that would be better dealt with in the community, and then are being readmitted to the hospital only days after leaving. To address these issues and improve efficient continuity of care, the Health Links program provides a new model of care at the clinical level in Ontario. In this model all of the patient's health service providers in the community, including primary care, hospital, and community care, work together to create a coordinated care plan for the patient. The initial focus of Health Links is on highcost users.

Health Links, and primary care as a whole, require comprehensive data analysis to effectively support patients and providers. SHIIP is a portal-based technology solution that enhances individual patient care while providing real-time feedback and summarized data to help plan care. The primary objective of SHIIP is to develop an Integrated Portal with core functionalities that will facilitate the sharing of information and enable person-centred care coordination. SHIIP aims to assists the success of Health Links by providing consistent maintenance and sharing of patient records, timely communication and collaboration between a patient's multiple health care providers, and removing physical barriers through the virtualization of care processes. SHIIP is designed to identify and assist in the delivery of care for complex/high needs patients, and will facilitate reporting, performance monitoring and quality improvement efforts. Some of the anticipated benefits of SHIIP include: enhanced patient experience, reduced workflow duplication, improved access to information at point of care, more efficient clinical documentation, and improved health outcomes. Ultimately, SHIIP helps to improve access and quality of healthcare, and consequently health equity, especially for complex/high-needs patients.

\section{Methods}

SHIIP provides the technology to enable collaborative, multiagency care processes. The SHIIP system collects patient demographic and clinical data from hospital databases. Most of the data is captured from its sources in real-time or near real-time, with an emphasis on unidirectional flow of information from hospitals to primary care providers. Through this process, primary care providers are supplied with timely access to various clinical data. Algorithms developed in the system use the information collected to identify complex/high needs patients. SHIIP also uses the collected data to notify, in-real time, primary care providers, Health Links coordinators, and other health care providers of patient encounters and transitions, and enables tracking of patients within the health system. In addition, as more data becomes available after patient discharge, the complex patient identification algorithms are updated to continuously enrich the patient's profile. Expansion of SHIIP to additional health care providers is scheduled for future phases.

In order for end-users to access the information collected, SHIIP has a single centralized web interface for all primary care providers in the SELHIN. The web-based graphical user interface allows primary care providers to view their patients' Emergency Department, Acute Inpatient, and Outpatient clinic visits and related information. The interface highlights key patient characteristics useful to primary care providers, such as: current admissions, visit history, chief complaints, complex/high needs flags, interventions, patient flow and other risk factors. This functionality enhances awareness of patient needs and improves communication among different health care providers to enable better care coordination planning and more efficient delivery of health care.

\section{Results}

SHIIP is currently in use in the SELHIN (a population of approximately 500,000 people), with an evaluation anticipated in 2 years.

\section{Conclusions}

The use of SHIIP will improve the access to and quality of care, especially for complex/high-needs patients. These improvements, which will enable person-centered care coordination, will be achieved through the consistent maintenance and sharing of patient records between health care providers.

\section{Keywords}

portal; primary care; real-time 